\title{
Event-based simulation of interference with alternatingly blocked particle sources 1
}

\author{
K. Michielsen*, S. Mohanty*, L. Arnold* and H. De Raedt ${ }^{\dagger}$ \\ * Institute for Advanced Simulation, Jülich Supercomputing Centre, \\ Research Centre Jülich, D-52425 Jülich, Germany \\ ${ }^{\dagger}$ Department of Applied Physics, Zernike Institute for Advanced Materials, \\ University of Groningen, Nijenborgh 4, NL-9747 AG Groningen, The Netherlands
}

\begin{abstract}
We analyze the predictions of an event-based corpuscular model for interference in the case of two-beam interference experiments in which the two sources are alternatingly blocked. We show that such experiments may be used to test specific predictions of the corpuscular model.
\end{abstract}

Keywords: Two-slit interference, quantum theory, event-based corpuscular model

PACS: 03.65.Ud, 03.65.Ta

\section{INTRODUCTION}

Two slit interference experiments have been used to probe fundamental properties of light and matter for over two centuries. While Young's original experiment helped establish the wave theory of light [1], variants of the experiment over the years have helped revealing fascinating wavelike properties of material entities from electrons $[2,3]$ to the comparatively humongous bucky balls [4].

One interesting variant involves a very dim source so that on average only one photon is emitted by the source at any time. Photons are also detected one at a time, as distinctly unwavelike "clicks" at the detector, at seemingly random positions [5]. Yet, when a large number of these clicks have been collected, their locations collectively form precisely the same kind of interference pattern as would be expected from a wave interacting with the slits. Such experiments have helped the development of ideas on concepts such as wave-particle duality in quantum mechanics.

And yet, interference in itself does not entail that the participants be waves. So long as a concept of an oscillating phase can be associated with individual particles, even a particulate model can produce interference. Feynman's path integral description is an example [6]. In this paper, we present a toy model for photon interference effects which are created by the detector. This is in contrast to the standard picture where each particle interferes with itself and the detector passively detects the result. The inclusive results (averages) from the model about interference are very similar to those obtained from quantum mechanics, even though each particle has a definite trajectory through one or the other slit to the detector. We also discuss more detailed experiments that may be able

\footnotetext{
${ }^{1}$ FPP6 - Foundations of Probability and Physics 6, edited by A. Khrennikov et al., (AIP Conference Proceedings, Melville and New York, in press)
} 
to distinguish between predictions of this model and standard calculations.

\section{MODEL AND METHODS}

In our model [7, 8], which we will refer to as the "Event Based Corpuscular Model" (EBCM), each photon carries a $2 \mathrm{D}$ vector $\vec{e}$ which oscillates in time with the photon's frequency according to

$$
\vec{e}=(\cos \omega t, \sin \omega t) .
$$

The two slits are modelled as a pair of coherent sources stochastically emitting photons isotropically. We imagine that the detector screen consists of an array of detector elements (pixels) which react to the incoming photons in the following way. The detector element has a vector property (e.g. polarisation) which responds to the photon's vector $\vec{e}$ as:

$$
\vec{p}_{i}=\gamma \vec{p}_{i-1}+(1-\gamma) \vec{e}_{i}, \quad 0<\gamma<1 .
$$

In other words, $\vec{p}_{i}$ responds to the incoming photon vector $\vec{e}_{i}$, but keeps a memory of its previous state. The parameter $\gamma$ controls how slowly the detector reacts to one individual incident photon. We assume that the detector element "clicks" when the length of the vector $\vec{p}$ reaches a certain threshold.

Simulated photons arrive at one of the detector elements and change its state according to Eq. (2). Every once in a while, the vector length at a detector element reaches the click threshold and an event is recorded. We then perform a simulation and collect statistics of the click counts along the detector elements [7,8].

Several sets of simulations are performed to study the effect of the memory parameter $\gamma$ and another parameter $N$. The parameter $N$ is used to examine in detail the effects of prior knowledge of the path of each photon. In the simulations each source is allowed to emit $N$ photons while the other source is blocked, and then the states of the two sources (blocked/unblocked) is interchanged.

\section{RESULTS}

Fig. 1(a) shows the two slit setup for which we show our results, and compare with the standard optics calculation. The intensity $I(\theta)$ observed at an angle $\theta$ far away from the two slits is given by

$$
I(\theta)=A\left(\frac{\sin (a \pi \sin \theta / \lambda)}{a \pi \sin \theta / \lambda}\right)^{2} \cos ^{2}(\pi d \sin \theta / \lambda),
$$

where $d$ is the separation between the centres of the slits, $a$ the width of the slits and $\lambda$ the wavelength.

For a basic test, we compare in Fig. 1(b) the interference result given by Eq. (3) and the one obtained from simulations with EBCM. For each event in simulations with EBCM, an emission point along the lengths of one of the two slits, as well as a direction of travel for the photon are chosen at random (uniformly distributed). Like in experiments 

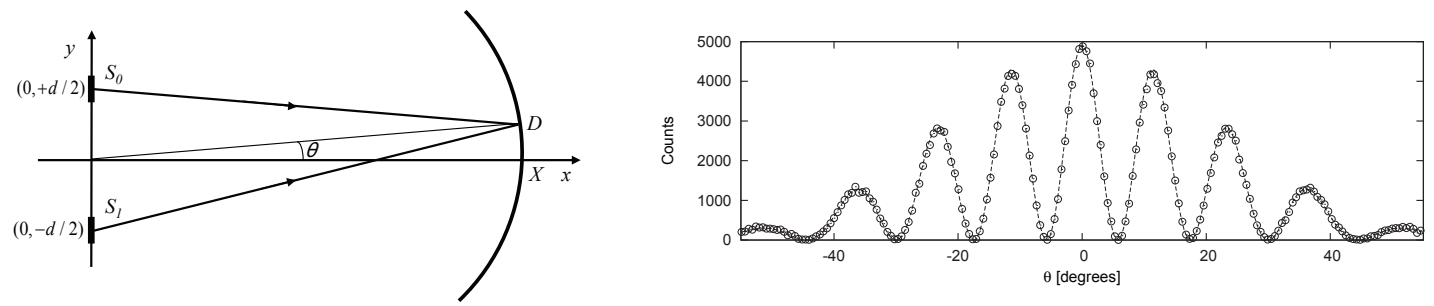

FIGURE 1. a) Basic setup for all comparisons. The distance between the slits $S_{1}$ and $S_{2}$ is $d=5 \lambda$, while the slits have a width $a=\lambda$ with $\lambda=670 \mathrm{~nm}$. The circular detector surface is assumed to be at a large distance $X=0.05 \mathrm{~mm}$ from the sources. (b) Comparison of general interference results from EBCM simulations (circles) and the regular optics calculation (solid line).

with low intensity, detector pixels in simulations record events apparently at random locations, but the interference pattern in Fig. 1(b) builds up over time, in complete agreement with Eq. (3). The EBCM therefore passes the first basic test as a model for interference with individual particles.

In standard two slit interference calculations using quantum mechanics, the wave function of the particles interacts with the two slits, and produces interference in the amplitude at the detector. The probability of a particle arriving at a certain location on the detector then turns out to be just like in classical optics. The particles do not produce interference patterns due to their mutual interactions, but rather the wave function of individual particles interferes with itself after being filtered by the two slits. Only after a large number of events have been measured can the probabilities be compared with the calculations. On the surface, the EBCM behaves similarly. However, one can think of more detailed experiments to distinguish it from standard quantum mechanical calculations.

In particular, in EBCM, we can have knowledge of which slit the photons travel through, and yet have interference. In quantum mechanics, the wave function needs to have a non-zero amplitude at the location of both the slits in order to produce interference. Experiments can be designed for which the predictions from EBCM differ from quantum mechanics. Consider a variant of the two slit experiment in Fig. 1, in which the slits open and close periodically so that at any time there is only one slit open. For simplicity, one can model the situation like this: the sources $S_{1}$ and $S_{2}$ alternatingly emit groups of $N$ photons each, until a total of $M$ photons have been emitted.

In Fig. 2, we show EBCM results for slit switching experiments of different $N$. Evidently, except at very large values of $N$, the interference pattern remains unchanged. In the simplest calculation with quantum mechanics, the intensity pattern at the detector for this situation should be the sum of two single slit patterns, as the particles pass through one or the other slit, and never through both.

The interference patterns seen in EBCM are a result of memory effects in the detection units of the experimental setup. This is parametrised by $\gamma$ in Eq. 2. In Fig. 3, we show EBCM results for a smaller $\gamma=0.9$ compared to Fig. 2. By comparing the figures for the corresponding $N$ values, we see that smaller $\gamma$ value smooths out the interference pattern in EBCM. 

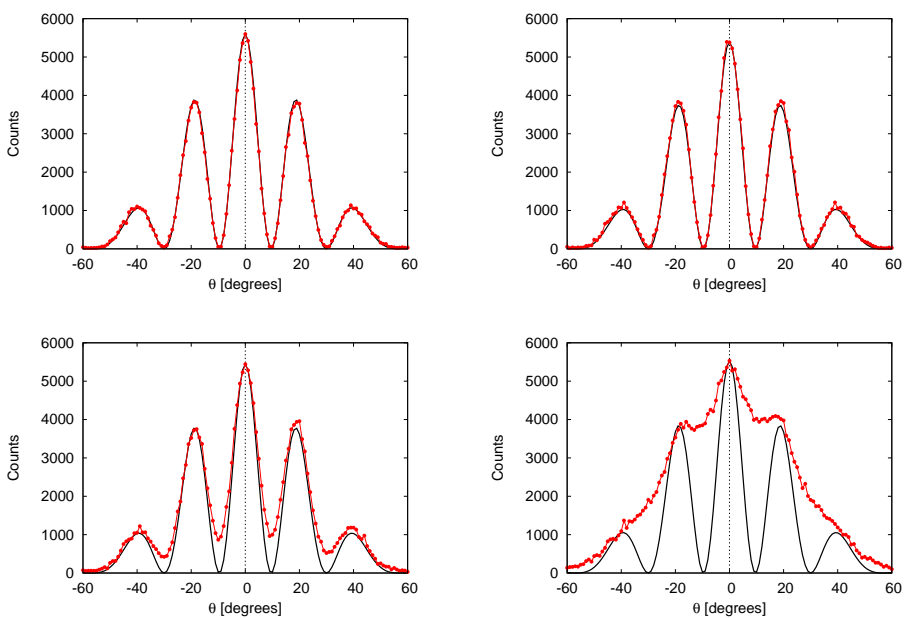

FIGURE 2. EBCM results for slit switching experiments. Each source $S_{1}$ and $S_{2}$ in Fig. 1 emits $N$ photons after which it is switched off and the other source is switched on. From top left to bottom right (row first) we show the EBCM simulation results (red dots) for $N=1, N=1000, N=100000$ and $N=500000$. For all plots here, $\gamma=0.999$, and the total number of emitted photons $M=1000000$. The solid line represents the intensity given in Eq. (2)
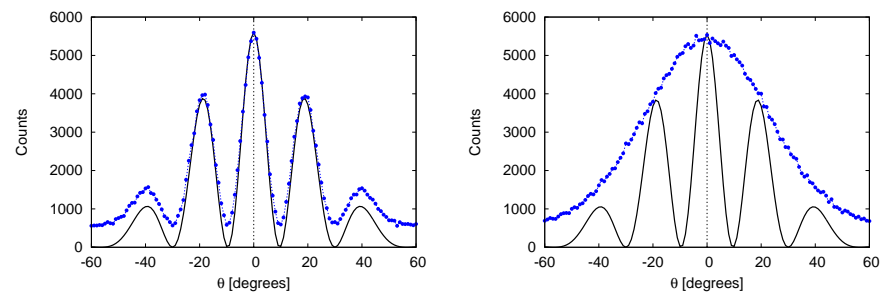

FIGURE 3. Same as Fig. 2 for $\gamma=0.9$. Shorter memory, i.e., smaller $\gamma$ leads to a smoothening of the interference pattern in EBCM compared to the results shown in Fig. 2. Left: $N=1$. Right: $N=500000$.

\section{DISCUSSION}

In its present formulation, the event based corpuscular model discussed in this paper, is not a general physical concept with which to understand the physical world. It might not be applicable to electrons, as the arrival of the electrons at the detector can be measured by the electric charge they carry to the individual detector elements. In that case, the detector elements react to each individual incident electron with a "click" and the threshold behaviour of the modelled detection elements becomes superfluous. Hence, application of the EBCM discussed in this paper to a similar experiment with electrons would produce no interference at all. Even in the case of photons, the ECBM is not an attempt to tell us what the photons "actually do". The EBCM is just a convenient model to describe the observations in standard two slit experiments.

Nevertheless, sticking to the model idea that interference arises due to state memory in the experimental setup, there may be measurable consequences that are different from quantum mechanics. One kind of such experiments would be those in which only one 
of the two slits is available to the particles at a time. In EBCM the results depend on the switching frequency in relation to the photon emission rate.

Although we are not aware of any experiment that precisely tests the above scenario, one experimental study in which only one slit was available to each photon [9] produced intriguing results. In that study, an opaque barrier, all the way from the laser source to the obstacle between the two slits, was used to make sure that photons had one or the other slit available to them. The interference pattern observed was nevertheless essentially unchanged despite the barrier. We are, however, not aware of any follow-up work on that study.

As a final note, we observe that the model presented here is different from the "particles with clocks" picture in Feynman's delightful explanation of the path integral description of light propagation [6]. The path integral formulation is essentially quantum mechanical: the amplitude was obtained by summing over all possible paths. It does not depend on detector memory. In the switching slits experiments proposed above, it leads to the same predictions as quantum mechanics.

\section{SUMMARY}

We have discussed an event based corpuscular model for interference of photons in a two slit experiment. In this model, interference arises due to memory effects in the experimental setup. For the most basic setup of the two slit experiment, the model has identical predictions compared to quantum mechanics. But it differs from quantum mechanics in predictions about modified experiments in which only one of the two slits is available to the particles at any time, and predicts a slow degradation of the interference signal as the switching between the frames is made progressively slower in relation to the rate of photon emission. More careful calculations using quantum mechanics as well as new experiments using today's technology could clarify the picture.

\section{REFERENCES}

1. T. Young, Philos. Trans. R. Soc. London 92, 12-48 (1802).

2. P. G. Merli, G. F. Missiroli, and G. Pozzi, Am. J. Phys. 44, 306-307 (1976).

3. A. Tonomura, J. Endo, T. Matsuda, T. Kawasaki, and H. Ezawa, Am. J. Phys. 57, 117-120 (1989).

4. M. Arndt, O. Nairz, J. Vos-Andreae, C. Keller, G. van der Zouw, and A. Zeilinger, Nature 401, 680-682 (1999).

5. V. Jacques, E. Wu, T. Toury, F. Treussart, A. Aspect, P. Grangier, and J.-F. Roch, Eur. Phys. J. D 35, 561-565 (2005).

6. R. Feynman, QED: The strange theory of light and matter, Universities Press, 1985.

7. F. Jin, S. Yuan, H. De Raedt, K. Michielsen, and S. Miyashita, J. Phys. Soc. Jpn. 79, 074401 (2010).

8. K. Michielsen, F. Jin, and H. De Raedt, J. Comp. Theor. Nanosci. 8, 1052 - 1080 (2011).

9. D. L. Alkon, Biophysical Journal 80, 2056-2061 (2001). 